\title{
Bronchography in the assessment of patients with lung collapse for endoscopic laser therapy
}

\author{
P J M George, M C Pearson, D Edwards, R M Rudd, M R Hetzel
}

\begin{abstract}
In an attempt to improve selection of patients and the efficacy of endoscopic laser treatment, a bronchographic technique has been developed for patients with tumours causing complete endobronchial obstruction. This technique has shown patent distal airways in 16 out of 17 patients with a collapsed lung or lobe. These airways were abnormally dilated in each case, suggesting bronchiectasis. In one patient the appearances of bronchiectatasis were sufficiently severe to decide against attempting treatment. Treatment was not attempted in another patient as a large cavity was seen within the collapsed lung and this was thought to carry a risk of postoperative infection and haemorrhage. Treatment with a neodymium YAG laser under general anaesthesia successfully recanalised the airway in 12 of the 15 remaining patients and was associated with a substantial reduction in breathlessness. The procedure was abandoned prematurely in one patient because of life threatening haemorrhage. In the remaining two patients in whom treatment was unsuccessful bronchography had suggested very extensive endobronchial obstruc-
\end{abstract} tion. Spirometry and radionuclide lung scans were performed before and after treatment in eight patients treated successfully and showed significant improvements. Four patients were investigated within two weeks of lung re-expansion by repeat bronchography (three patients) or computed tomography (one patient); in each case the calibre of the airways had returned almost to normal. Thus the radiological demonstration of bronchial dilatation in a collapsed lung does not necessarily imply a diagnosis of irreversible bronchiectasis and should not be regarded as a contraindication to treatment. It is concluded that preoperative bronchography provides reliable data on the extent of tumour, the patency of the distal airways, and presence of extensive cavitation. This information should facilitate successful laser treatment.

The neodymium YAG laser provides a convenient method of relieving large airway obstruction in patients with advanced lung cancer. The thermal effects of the laser may be used to resect and cauterise intraluminal tumour and, in appropriately selected cases, to palliate breathlessness and other obstructive symptoms. Treatment produces symptomatic improvement in about $70-80 \%$ patients with partial airway obstruction but is considerably less successful in patients with tumours causing complete endobronchial obstruction and atelactasis. $^{12}$ For example, successful reexpansion was achieved in only five of 17 patients with a collapsed lung or lobe. ${ }^{1}$

There are several possible explanations for this lack of success in treating patients with complete endobronchial obstruction. Firstly, the operator may have difficulty in selecting the correct plane of dissection through the tumour because the normal anatomical landmarks are lost. Secondly, the operator has no knowledge of the amount of tumour that must be removed before he can reach patent distal airways. Treatments may therefore be abandoned prematurely in patients with salvageable lungs. Finally, the operator does not know whether patent distal airways exist beyond the tumour. The tumour may extend into the periphery of the lung and be associated with extensive damage, so that patients with non-viable lungs may be subjected needlessly to the hazards of laser treatment.

In an attempt to overcome these problems, we have developed a bronchographic technique to show the endobronchial extent of the tumour and patency of the distal airways before laser treatment is attempted. The technique and our results are described in this paper.

\section{Patients and methods}

PATIENTS

We studied 17 patients (10 males and seven females) with inoperable bronchial malignancy. The average age was 66 with a range of 43-78 years. All patients were severely limited by breathlessness and their airways were obstructed predominantly by intraluminal tumour. Obstruction was within a main bronchus in each case; 16 patients had collapse of a lung and one collapse of a lower lobe. Serial chest radiographs indicated that collapse had been present for at least one month. The histological types of the tumours were: squamous cell carcinoma (10 patients), large cell carcinoma (5), small cell carcinoma (1), and undifferentiated carcinoma (1). Seven patients had not received any previous treat-
Address for reprint requests: Dr P J M George, London Chest Hospital, London E2 Accepted 25 April 1990 


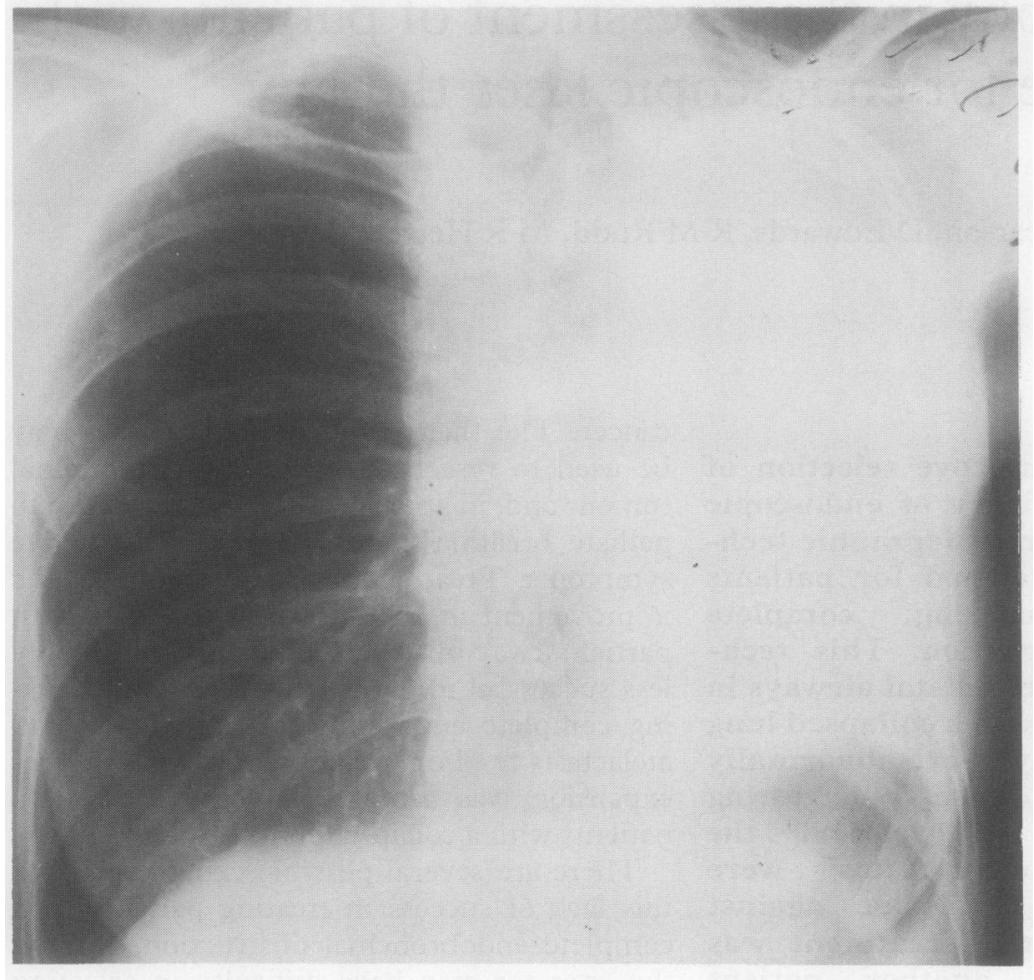

Figure 1 A collapsed left lung ( $a$ ) caused by a tumour occluding the left main bronchus. The tip of the catheter has been advanced into the lower lobe and radio-opaque contrast has been injected to reveal patent distal airways; the proximal and distal margins of the tumour are also well demonstrated $(b)$. Laser treatment successfully restored an airway to the lower lobe, which then reexpanded.

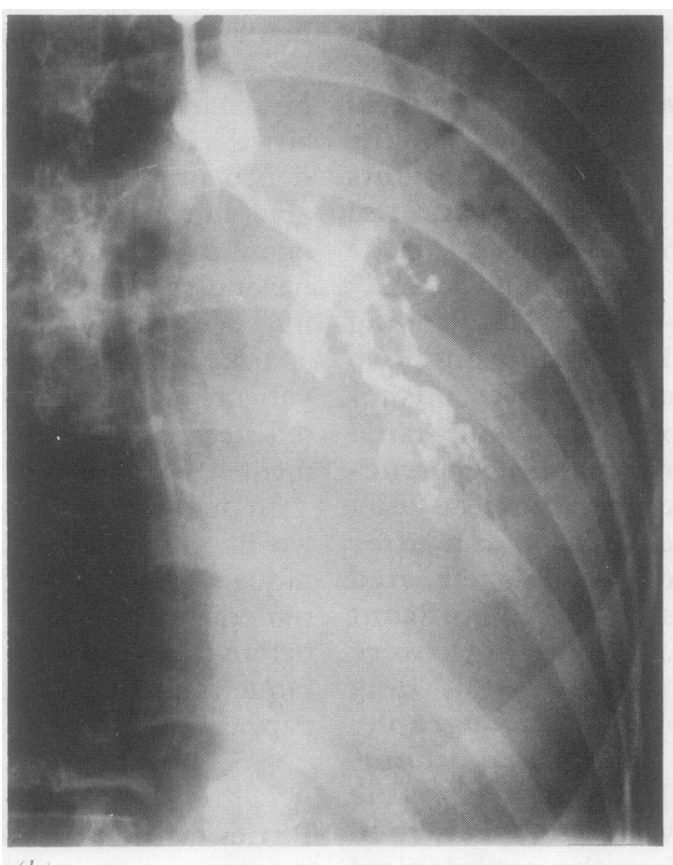

ment and seven had undergone radiotherapy, two chemotherapy and radiotherapy, and one chemotherapy alone.

We studied two additional patients with squamous cell carcinomas causing obstruction of a main bronchus. Although both had presented with a collapsed lung, their lungs had re-expanded at the time of admission to hospital. Re-expansion occurred spontaneously in one patient and after a course of palliative radiotherapy in the other. Serial chest radiographs indicated that the interval between re-expansion and bronchography was no greater than two weeks.
BRONCHOGRAPHY

After premedication with intramuscular injections of papavaretum and atropine, fibreoptic bronchoscopy was performed under local anaesthesia with topical lignocaine. If necessary, additional sedation was produced with up to $10 \mathrm{mg}$ intravenous diazepam. A multipurpose angiographic catheter (Cordis, FG 7), containing a J guide wire, was passed through the biopsy channel of the bronchoscope and directed through the tumour under radiographic screening. The J guide wire was advanced beyond the tip of the catheter to allow the catheter to pass into the distal airways with minimal trauma.

When the catheter had been advanced 3$4 \mathrm{~cm}$ beyond the proximal border of the tumour, the guide wire was removed and water soluble radio-opaque contrast (Omnipaque) was injected through the catheter under screen control. The air within the catheter, which was expelled, outlined the distal airways, which were then opacified with contrast. If distal airways were not seen, the guide wire was replaced and the catheter advanced. The procedure was repeated until bronchi were identified. The catheter was then slowly withdrawn while continuing to inject contrast in order to demonstrate the proximal and distal margins of the tumour in addition to the bronchial tree beyond (fig 1).

LASER TREATMENT

Treatments were given with neodymium YAG lasers operating at a wavelength of either $1.064 \mu \mathrm{m}$ (Fiberlase 100, Pilkington Medical Systems; University College Hospital) or $1.32 \mu \mathrm{m}$ (MBB-Medizintechnic; London Chest Hospital). Treatment with the two lasers was essentially the same; both used a combination of rigid and flexible bronchoscopes, with general anaesthesia produced by intravenous agents. ${ }^{3}$ For treatment with the $1.064 \mu \mathrm{m}$ laser, however, we used power settings of 40-70 watts in pulses of up to two seconds, but with the $1.32 \mu \mathrm{m}$ laser powers of 10-20 watts in pulses of up to one second.

\section{PREOPERATIVE AND POSTOPERATIVE}

ASSESSMENTS

Lung re-expansion was assessed in all patients with chest radiographs. Breathlessness was graded on the basis of the Medical Research Council (MRC) questionnaire. ${ }^{4}$ Measurements of peak expiratory flow (PEF), forced expiratory volume in one second $\left(F E V_{1}\right)$, and forced vital capacity (FVC) were obtained whenever possible.

We also attempted to evaluate changes in ventilation and perfusion within the treated lung by comparing preoperative and postoperative radionuclide lung scans. Perfusion was assessed by using technetium-99m labelled human albumin macroaggregates, and ventilation with either krypton-81m gas (University College Hospital) or ${ }^{99 \mathrm{~m}} \mathrm{~T}$ labelled DTPA aerosols (London Chest Hospital). Anterior and posterior views were obtained and the number of counts was averaged by taking the geometric mean counts from the 
two views. Ventilation and perfusion were quantified by expressing the mean number of counts in the treated lung as a percentage of the sum of the counts in both lungs to produce a fractional score. Full details of this method have been described. ${ }^{5}$ Patients whose airways had not been recanalised were not asked to repeat these tests after the procedure.

\section{ANALYSIS}

Mean values for breathlessness score (MRC grade) were compared before and after treatment by Wilcoxon's matched pairs signed rank test. Student's paired $t$ test was used to assess lung function $\left(\mathrm{FEV}_{1}, \mathrm{FVC}, \mathrm{PEF}\right)$ and fractional scores of ventilation and perfusion before and after treatment.

Figure 2 Preoperative bronchographs from two patients who were not treated with the laser. (a) Bronchograph in a patient with tumour occluding the left main bronchus, showing severe dilatation of the airways beyond the obstruction. The "bronchiectatic" appearances were so severe that it was decided not to attempt laser treatment; more recent experience, however, has shown that these appearances may resolve after laser treatment (see fig 4). (b) Bronchograph in a patient with a collapsed right lung, showing a large cavity within the upper lobe. Previous experience has shown that these patients are prone to severe infection and haemorrhage after the treatment.
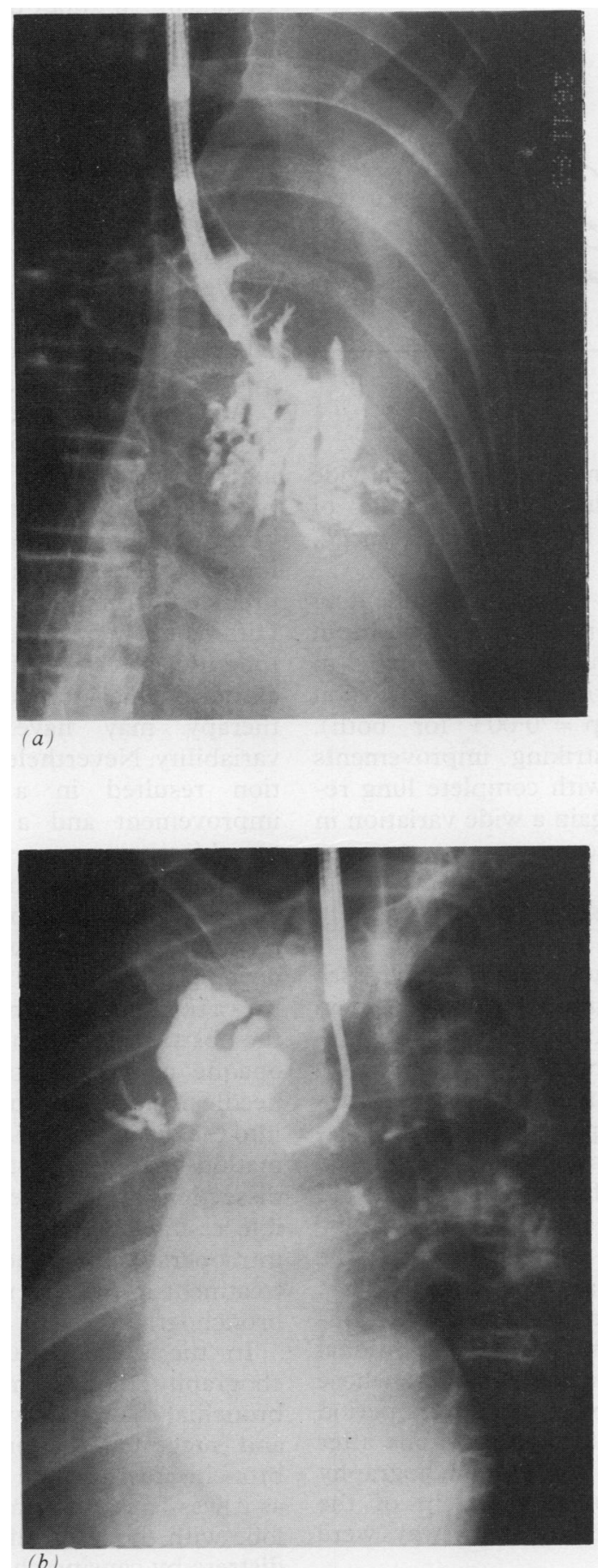

\section{AIRWAYS}

In patients with a collapsed lung postoperative chest radiographs showed complete re-expansion in six and partial re-expansion in five. $\mathrm{Re}-$ expansion was also obtained in the patient with a collapsed lobe. In each case breathlessness was reduced to such an extent that the MRC grade in the 12 patients improved from a mean value of 4.4 to $2 \cdot 8(\mathrm{p}=0.002)$.

Respiratory function was assessed before and after treatment in eight patients (table). There was a significant increase in mean values of $\mathrm{FEV}_{1}$ and FVC ( $\mathrm{p}=0.05$ and 0.005$)$ but no change in PEF. The most striking improvements were observed in patients with

Mean (SD) peak expiratory flow (PEF), forced expiratory volume in one second $\left(F E V_{1}\right)$, and forced vital capacity (FVC) before and after laser treatment in eight patients in whom a lung or lobe was re-expanded

\begin{tabular}{lcc}
\hline & Before & After \\
\hline PEF (1/min) & $191(48)$ & $188(78)$ \\
FEV $_{1}(1)$ & $1.26(0.42)$ & $1.49(0.55)^{\star}$ \\
FVC $(1)$ & $1.91(0.79)$ & $2.42(0.95)^{\star \star}$ \\
\hline
\end{tabular}

${ }^{\star} \mathrm{p}=0.05 ;{ }^{\star \star} \mathrm{p}=0.005$ (paired Student's $t$ test). 
Figure 3 Fractional scores of (a) ventilation and (b) perfusion before and after treatment in eight patients whose lungs or lobes were re-expanded with the laser. Mean scores are shown by solid squares. Seven patients were treated for complete collapse of a lung and their pretreatment scores of ventilation represent background counts.

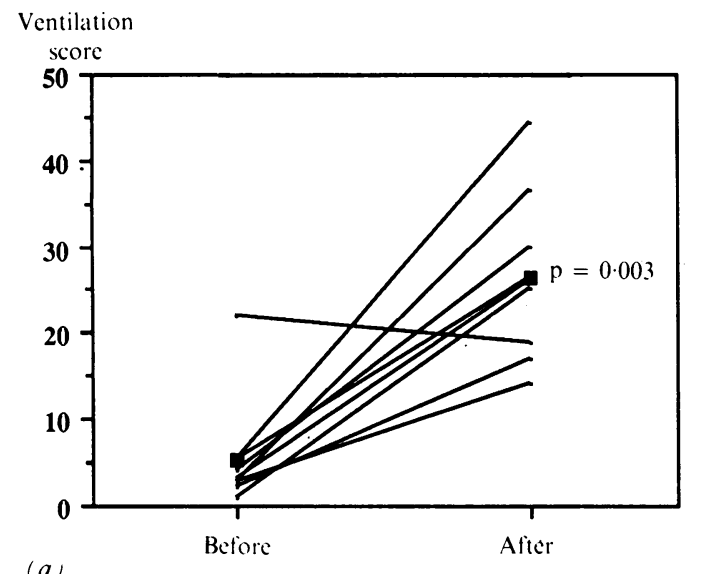

(a)

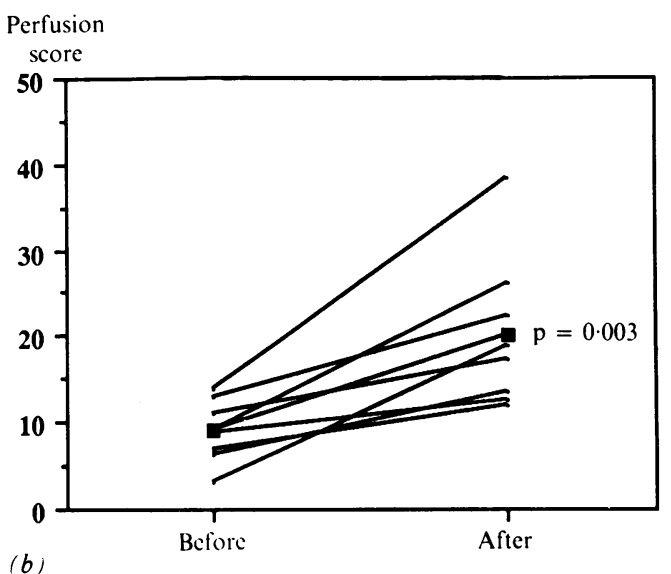

complete lung re-expansion; there was a wide variation, however, in the magnitude of change, the increase in FVC ranging from $7 \%$ to $69^{\circ}{ }_{0}$.

The results obtained with radionuclide lung scans are summarised in figure 3 . The mean fractional score of ventilation rose from 5.4 to 26.4 , and the mean fractional score of perfusion from 8.9 to $20.1 \quad(p=0.003$ for both). Although the most striking improvements were seen in patients with complete lung reexpansion, there was again a wide variation in the magnitude of change.

\section{ASSESSMENT OF THE REVERSIBILITY OF BRONCHIAL DILATATION}

Repeat bronchography and computerised tomography Bronchography was repeated in three patients within two weeks of lung reexpansion. In all cases the calibre of the airways had returned to almost normal (fig 4). In a fourth patient the re-expanded lung was assessed by computed tomography. This showed only slight thickening of the bronchial wall and dilatation of the airways, indicating considerable improvement by comparison with the preoperative bronchogram.

Bronchography after recent expansion Bronchography was performed in two additional patients with endobronchial tumours, whose lungs had recently re-expanded after a period of collapse-one spontaneously and one after radiotherapy. In both cases bronchography delineated the endobronchial extent of the tumour but showed that the distal airways were of normal calibre.

\section{Discussion}

In an attempt to evaluate patients with a collapsed lung, we initially performed computed tomography but found that the scans could not define bronchial anatomy within the area of the collapse. Our experience with preoperative bronchography has been more encouraging, however, as it has provided reliable information on the extent of tumour and patency of the distal airways. It has also enabled us to avoid the potential hazards of infection and haemorrhage, which we have previously encountered after recanalising cavitated lung. The technique has been easy to perform and well tolerated, and appears to be safe.

In addition to its value as a preoperative screening procedure, bronchography may improve the chances of achieving successful reexpansion. Without the knowledge that patent distal airways existed beyond the tumour, treatment might have been abandoned prematurely and would not have been repeated in three patients in this series whose lungs subsequently re-expanded. The patients with complete endobronchial obstruction who were selected for bronchography had longstanding collapse and thus represented the most challenging group of patients to be treated. Successful recanalisation in 12 of the 15 patients compares favourably with previously published figures of 5 of $17,{ }^{1} 11$ of $23,{ }^{2}$ and 7 of $11 .^{6}$

Although radiological re-expansion provides a convenient end point, it represents a poor index of functional improvement. In the present study spirometry and radionuclide lung scanning revealed a wide range of improvement, which could not have been predicted from the plain chest radiograph. Differences in postoperative airway calibre and the inconsistent effects on the lung of extrabronchial tumour and previous radiotherapy may have contributed to this variability. Nevertheless, successful recanalisation resulted in a significant functional improvement and a valuable reduction in breathlessness.

Laser recanalisation in patients with a collapsed lung may be further helped by the technique of intraoperative bronchography, developed by Joyner et al. ${ }^{7}$ Their technique uses a transbronchial biopsy needle to perforate the obstructing tumour and then inject radioopaque contrast distally. The angle of the needle is adjusted until contrast flows freely into the distal airway, thereby providing information that guides the operator's plane of dissection through the tumour. The best possible results therefore may be obtained when intraoperative bronchography is used to guide treatment in patients selected by preoperative bronchography.

In the present study preoperative bronchography consistently showed abnormal bronchial dilatation within the collapsed lung and suggested the presence of radiographic bronchiectasis. These findings were of concern as it was feared that re-expansion of a lung or lobe with the laser might add to the patient's distress by causing chronic productive cough. 
Figure 4 Example of reversible bronchial

dilatation. Collapsed right

lung $(a)$, caused by a

tumour occluding the right main bronchus.

Preoperative bronchography ( $b$ ) showed tumour extending from the orifice of the right main bronchus to the intermediate

bronchus. The middle and lower lobe bronchi were patent but dilated. The upper lobe bronchus could not be identified.

Endoscopic laser resection successfully recanalised an airway to the intermediate bronchus but not to the upper lobe, and resulted in re-expansion of the middle and lower lobes ( $\mathrm{c}$ ).

Bronchography two weeks later showed that the

calibre of the right middle and lower lobe bronchi had improved substantially /d and $e$.
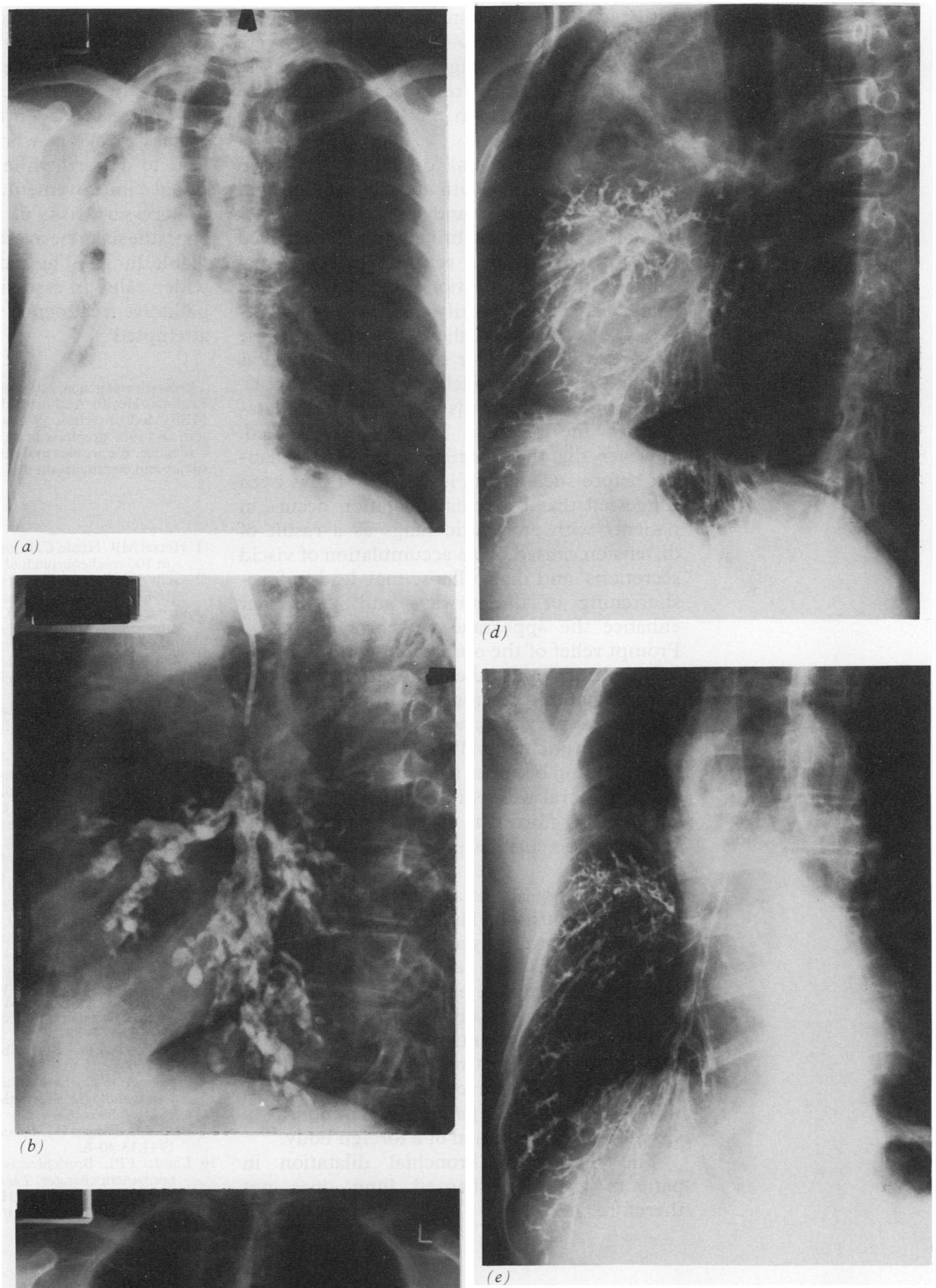

Furthermore, our previous experience has shown that patients whose lungs or lobes are successfully expanded are particularly at risk of developing pneumonia after the procedure, occasionally with fatal outcome. ${ }^{1}$

Joyner et al do not say whether any of their patients had bronchial dilatation. ${ }^{7}$ Nevertheless, the finding of bronchial dilatation is well known in atelectatic lungs obstructed by endobronchial tumours ${ }^{8}$ and inhaled foreign bodies. ${ }^{9}$ In the present study the finding of normal bronchial calibre in two patients whose lungs had re-expanded after a period of collapse and of reversible bronchial dilatation (fig 4) in four patients suggests that the dilatation is not necessarily permanent and may be 
reversed when the lung or lobe re-expands. The decision not to treat the patient with severe bronchial dilatation (fig $2 a$ ) was taken before we had observed this reversibility and would not have been made in the light of our more recent experience.

Reversible bronchial dilatation has been observed in patients with atelectasis associated with viscid secretions and severe infection. ${ }^{10-16}$ In these cases repeat bronchography showed that the airways had returned to a normal calibre when the infection and secretions had been treated satisfactorily and the lung had reexpanded. Whether these cases and those presented in this paper should be regarded as genuine cases of bronchiectasis is debatable. The term bronchiectasis literally means dilatation of the bronchi; it has been argued, however, that the dilatation should be permanent before such a term is used. ${ }^{11}{ }^{17}$ It has been suggested that bronchial dilatation occurs in patients with atelectatic lungs as a result of distension caused by an accumulation of viscid secretions ${ }^{8}$ and that collapse may lead to foreshortening of the airways and so further enhance the appearances of bronchiectasis. ${ }^{7}$ Prompt relief of the obstruction with drainage of secretions and re-expansion of the lung should therefore restore a normal airway. If infection supervenes, however, it is suggested that the bronchial wall is weakened and the adjacent lung becomes fibrosed. ${ }^{8}$ The combination of mural weakening and traction by the surrounding fibrosis is thought to produce the characteristic changes of irreversible bronchiectasis.

If these suggestions are correct, it is clearly important to use the laser to attempt lung reexpansion as promptly as possible before infection and fibrosis have become established. The duration of complete endobronchial obstruction and of collapse could not be documented precisely in our patients but were probably in the region of one to two months. In Linton's series of 16 patients irreversible bronchiectasis was observed in four patients seven months to six years after inhalation of a foreign body. ${ }^{9}$

The finding of bronchial dilatation in patients with a collapsed lung does not therefore necessarily imply a diagnosis of irreversible bronchiectasis and should not be regarded as a contraindication to attempting laser treatment. We believe that bronchography is a valuable preoperative screening procedure as it identifies patients with patent distal airways in whom treatment is likely to lead to a worthwhile symptomatic and functional improvement. It also enables the unnecessary risks of treatment under general anaesthesia to be avoided in patients with nonviable lungs. The technique may also have a wider value in assessing patients before other palliative treatments, such as radiotherapy, are attempted.

This work was generously supported by the North East Thames Regional Health Authority, Pilkington Medical Systems, and MBB-Medizintechnic. We would like to thank the endoscopy staff and radiographers in our two hospitals for their technical staff and radiographers in our two hospitals for their technical assistance. We are also grateful to $M$
advice and comments on this work.

1 Hetzel MR, Nixon C, Edmonstone WM, et al. Laser therapy in 100 tracheobronchial tumours. Thorax 1985;40:341-5. 2 Gelb AF, Epstein JD. Neodymium-yttrium-garnet laser in lung cancer. Ann Thorac Surg 1987;43:164-7.

3 George PJM, Garrett CPO, Nixon C, et al. Laser treatment for tracheobronchial tumours: local or general anaesthesia? Thorax 1987;42:656-60.

4 Medical Research Council. Questionnaire on respiratory symptoms. London: Medical Research Council, 1966.

5 George PJM, Clarke G, Tolfree S, Garrett CPO, Hetzel MR. Changes in regional ventilation and perfusion of the lung after endoscopic laser treatment. Thorax 1990;45: lung aft 248 .

6 Haubinger K, Held E, Huber R. Endobronchial laser therapy-differential therapeutic use and clinical value. therapy-differential therapeutic

7 Joyner LR, Maran AG, Sarama R, Yakaboski A. Neodymium-YAG laser treatment of intrabronchial lesions: a new mapping technique via the flexible fibreoptic bronchoscope. Chest 1985;87:418-27.

8 Spencer H. Pathology of the lung. 4th ed. Vol 1. Oxford: Pergamon Press, 1985:131-65.

9 Linton JSA. Long-standing intrabronchial foreign bodies. Thorax 1957;12:164-70.

10 Findlay L. Atelectatic or compensatory bronchiectasis. Arch Dis Child 1935;10:61-84.

11 Jennings $\mathrm{GH}$. Re-expansion of the atelectatic lower lobe and disappearance of bronchiectasis. Br Med J 1937;ii:963-5.

12 Lander FPL, Davidson M. The aetiology of bronchiectasis (with special reference to atelectasis). Br J Radiol (with special refer

13 Fleischner FG. Reversible bronchiectasis. AJR 1941; 46:166-72.

14 Ogilvie AG. The natural history of bronchiectasis: a clinical roentgenologic and pathologic study. Arch Intern Med 1941;68:395-465.

15 Blades B, Dugan DJ. Pseudobronchiectasis. J Thorac Surg 1944;13:40-8.

16 Lander FPL. Bronchiectasis and atelectasis: temporary and permanent changes. Thorax 1946;1:198-210.

17 Hinshaw HC, Schmidt HW Some clinical problems in bronchiectasis. Dis Chest 1944;10:115-22. 